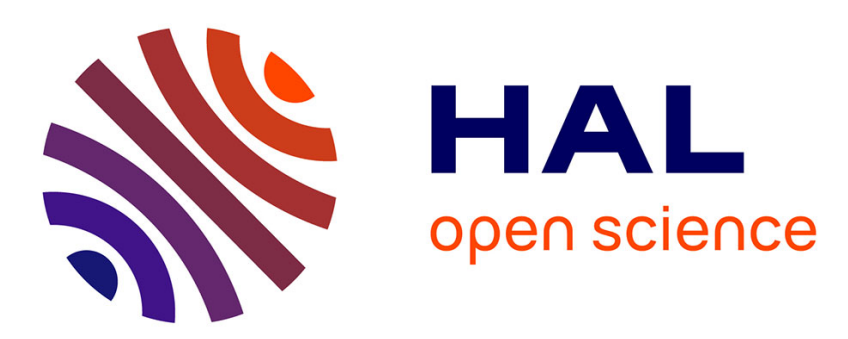

\title{
REVIEW OF THE ENERGY LIMITERS APPROACH TO MODELING FAILURE OF RUBBER
}

K y Volokh

\section{To cite this version:}

K y Volokh. REVIEW OF THE ENERGY LIMITERS APPROACH TO MODELING FAILURE OF RUBBER. Rubber Chemistry and Technology, 2013, 86, pp.470 - 487. 10.5254/rct.13.87948. hal-01573585

\section{HAL Id: hal-01573585 \\ https://hal.science/hal-01573585}

Submitted on 10 Aug 2017

HAL is a multi-disciplinary open access archive for the deposit and dissemination of scientific research documents, whether they are published or not. The documents may come from teaching and research institutions in France or abroad, or from public or private research centers.
L'archive ouverte pluridisciplinaire HAL, est destinée au dépôt et à la diffusion de documents scientifiques de niveau recherche, publiés ou non, émanant des établissements d'enseignement et de recherche français ou étrangers, des laboratoires publics ou privés.

\section{(c)(1)}

Distributed under a Creative Commons Attribution| 4.0 International License 


\title{
REVIEW OF THE ENERGY LIMITERS APPROACH TO MODELING FAILURE OF RUBBER
}

\author{
K. Y. VOLOKH \\ FACUlTy of CiVIL AND ENVIRONMENTAL ENGINEERING, TECHNION-ISRAEl INSTITUTE OF TeCHNOLOGy, \\ HAIFA 32000, ISRAEL
}

\begin{abstract}
Nonlinear theories of elasticity describe rubber deformation but not failure; however, in reality, rubbers do fail. In the present work, we review a new approach of energy limiters that allows for unifying hyperelasticity theories with failure descriptions, and we discuss results of this unification. First, we introduce the energy limiter concept, which allows the enforcement of failure descriptions in elasticity theories. The limiter provides the saturation value for the strain energy, hence indicating the maximal energy that may be stored and dissipated by an infinitesimal material volume. The limiter is a material constant that can be calibrated via macroscopic experiments. Second, we illustrate the new approach with examples in which failure initiation is predicted but its propagation is not tracked. Examples include the problems of crack initiation, cavity instability, and rupture of inflating membranes. In addition, the traditional strength-of-materials criteria are reassessed. Third, the theory is used for three-dimensional explicit finite element simulations of a high-velocity penetration of a stiff elastic body into a rubber plate. These simulations show that a high-velocity penetration of a flat projectile leads to a diffused nonlocal failure, which does not trigger the mesh sensitivity. To the contrary, a low-velocity penetration of a sharp projectile leads to a highly localized cracklike failure, which does trigger the mesh sensitivity. Calculation of the characteristic length of failure localization allows for setting the mesh size that provides regularization of the simulations. The fact that the calculation is based on results of solely macroscopic experiments is noteworthy.
\end{abstract}

\section{INTRODUCTION}

Failure of rubber and rubberlike materials is a fundamental issue. For example, the collapse of rubber tires because of crack propagation results in a more harsh loss of capital and life than airplane accidents. The correct modeling of failure can improve the design of rubberlike structures. In addition, it can help us to better understand the failure of rubberlike living tissues, improving their medical treatment.

Unfortunately, the analysis of mechanical behavior of rubberlike materials is difficult because of physical and geometrical nonlinearities. Ronald Rivlin ${ }^{1}$ made a great impact on the research in the mechanics of rubberlike materials by finding elegant analytical solutions to a number of problems, which were considered intractable. In parallel with the phenomenological approach of mechanics, a physical approach was developed that relied on the molecular structure of material. ${ }^{2,3}$ 
Although the physical approach appeals directly to the material structure, it is still somewhat phenomenological because models of molecular interactions and their statistics are approximate. Besides, the approach of continuum mechanics is, probably, more flexible and simple in fitting experimental data than the molecular considerations.

Although most studies on the mechanics of rubberlike materials have focused on the description of deformation, there are very few works on failure modeling. ${ }^{4-8}$ These studies aimed to clarify the asymptotic structure of the deformation and stress fields near the crack tip in various hyperelastic materials undergoing finite strains. Recently, Verron ${ }^{9}$ considered the so-called configurational mechanics as a tool to investigate the fracture of rubber. Another line of studying cracks in rubber stemmed from the physical observations of the influence of rubber viscosity on crack propagation. The works in this direction have been recently reviewed by Persson et al. ${ }^{10}$ It is interesting that the works considered in the cited review are based on linear elastic fracture mechanics (LEFM) and its linear viscoelasticity extension for rubbers (see also Gent ${ }^{11}$ ). Thus, both the material and geometrical nonlinearities are ignored. Such ignorance is probably justified when rubber is in the glassy state and it behaves like a hard, quasi-brittle material. The applicability of the linearized theory to the rubbery state of material is less evident. Nonlinear corrections to LEFM have been considered by Bouchbinder ${ }^{12}$ and Livne et al. ${ }^{13}$

New prospects for modeling failure of rubber and rubberlike materials are offered by the computer revolution, which took place in the last decades of the 20th century. The development of nonlinear finite element methods and their computer implementation allow tackling problems that we could have hardly imagined in the past. For example, interesting studies of cracks in rubberlike materials have been done by Marder. ${ }^{14,15}$ Nonetheless, the lack of studies of the failure of rubberlike materials is the main driving force for the present work.

\section{ELASTICITY WITH ENERGY LIMITERS}

The continuum mechanics approaches for modeling material failure can be provisionally divided into two groups: surface and bulk models.

The surface models, pioneered by Barenblatt, ${ }^{16}$ appear by the name of cohesive zone models (CZMs) in the modern literature. The cohesive zone is a surface in a bulk material where displacement discontinuities occur. Thus, continuum is enhanced with discontinuities. The latter requires an additional constitutive description. Equations relating normal and tangential displacement jumps across the cohesive surfaces with the proper tractions define a specific CZM. There are plenty of proposals for the cohesive constitutive equations. ${ }^{17-21}$ All of these models are constructed qualitatively as follows: tractions increase, reach a maximum, and then approach zero with the increasing separation. This scenario is in harmony with our intuitive understanding of the rupture process. Cohesive zones can be inside finite elements or along their boundaries..$^{22-24}$

Remarkably, the first models of bulk failure-continuum damage mechanics (CMD) proposed by Kachanov ${ }^{25}$ for analysis of the gradual failure accumulation and propagation in creep and fatigue appeared almost simultaneously with the cohesive zone approach. We should especially mention that CMD methods became very popular for modeling the Mullins effect in rubber. ${ }^{26-38}$ The need to describe the failure accumulation (i.e., evolution of the material microstructure) explains why damage mechanics is very similar to plasticity theories, including the internal damage variable (inelastic strain), the critical threshold condition (yield surface), and the damage evolution equation (flow rule). The subsequent development of the formalism of damage mechanics ${ }^{39}$ left its physical origin well behind the mathematical and computational techniques and eventually led to the use of damage mechanics for a description of any bulk failure. It should not be missed that bulk failure models should be regularized in problems in which failure tends to localize in narrow zones 


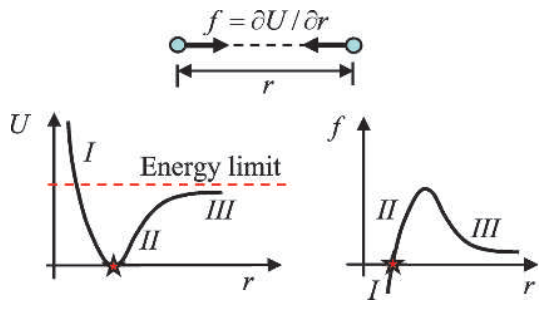

FIG. 1. — Particle interaction: I, repulsion; II, attraction; III, separation.

(e.g., cracks). A characteristic length should be introduced in the bulk models to limit the failure localization and avoid the mesh sensitivity of numerical calculations. ${ }^{40,41}$

We do not discuss the shortcomings of various continuum methods for modeling material failure. It is clear, however, that none of them is overall superior, and different methods are probably preferable for different problems.

Contrary to the methods of continuum mechanics for modeling material failure, the molecular mechanics and continuum-molecular methods ${ }^{42-44}$ use empirical potentials, which include a possibility of full molecular separation and rupture. The molecular and continuum-molecular methods are effective at small-length scales, whereas their use in macroscopic problems is computationally intensive.

As a very simple alternative to the mentioned approaches for modeling failure, here we propose the idea to limit the capacity of material to accumulate and dissipate the strain energy. The idea of limiting the strain energy density has deep physical roots because it introduces the average energy of molecular/atomic bonds in the continuum description of the bulk. To better comprehend the idea, let us consider an interaction of two particles, which can be molecules or molecular clusters (Figure 1).

The interaction undergoes repulsion, attraction, and separation. The separation starts at the limit point of the force-distance curve, shown in the right diagram of Figure 1. The limit point appears due to the existence of the energy limiter - the bond energy - for the particle potential, shown on the left diagram of Figure 1. In the case of solids, which comprise billions of particles, the average interparticle distance is measured by strain tensors and the average particle potential is measured by the strain energy function. Amazingly, in contrast to the particle interaction, the classical elasticity theory describing multiparticle systems does not include the energy limiter, which should be the average bond energy. Thus, the particle separation and, consequently, material failure is beyond the scope of traditional elasticity theories. ${ }^{46}$ However, the failure description can still be introduced in elasticity by analogy with the failure description in the particle interaction. The latter idea was put forward in a series of publications by Volokh. ${ }^{47-50}$

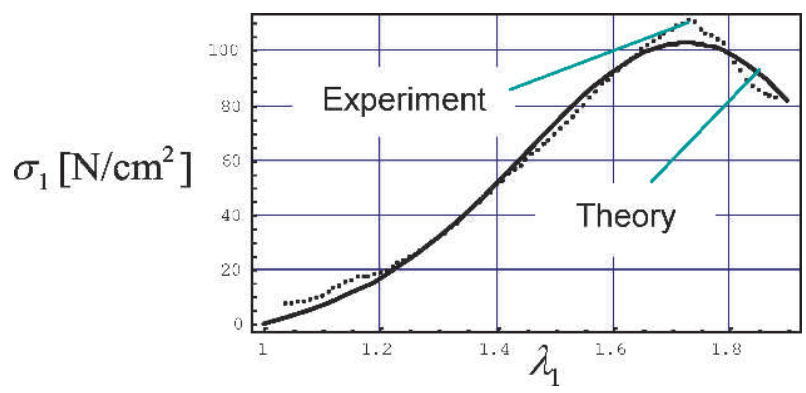

FIG. 2. - Cauchy stress versus stretch in the uniaxial tension of abdominal aortic aneurysm material (Volokh and Vorp ${ }^{45}$ ). 
A very simple yet general way to introduce the energy limiters is by using the following formula for the strain energy ${ }^{48}$ :

$$
\psi(\Phi, W)=\Phi-\Phi \exp \left(-\frac{W}{\Phi}\right)
$$

where $W$ is the strain energy of an intact (i.e., without failure) material and $\Phi$ is the energy limiter, which can also be interpreted in this case as the average bond energy or the failure energy. The latter provides the saturation value for the strain energy, indicating the maximum energy that can be dissipated by an infinitesimal material volume.

Eq. 1 has two limit cases. If the failure energy is infinite, $\Phi \rightarrow \infty$, then we have the classical hyperelastic material: $\psi(\infty, W) \rightarrow W$. If the failure energy is finite, then the increase of the strain energy is limited: $\psi(\Phi, \infty) \rightarrow \Phi$.

An example of the use of Eq. 1 can be found in Volokh and Vorp ${ }^{45}$ for the incompressible material of the abdominal aortic aneurysm, with the intact strain energy in the form $W=\alpha_{1}\left(\lambda_{1}^{2}+\lambda_{2}^{2}+\lambda_{3}^{2}-3\right)+\alpha_{2}\left(\lambda_{1}^{2}+\lambda_{2}^{2}+\lambda_{3}^{2}-3\right)^{2}, J=\lambda_{1} \lambda_{2} \lambda_{3}=1$, where $\lambda_{\mathrm{i}} \mathrm{s}$ are the principal stretches and material constants $\alpha_{1}=10.3 \mathrm{~N} / \mathrm{cm}^{2} ; \alpha_{2}=18.0 \mathrm{~N} / \mathrm{cm}^{2} ; \Phi=40.2 \mathrm{~N} / \mathrm{cm}^{2}$ were calibrated in the uniaxial tension test shown in Figure 2.

We emphasize that the energy limiter is calibrated in the macroscopic failure experiments.

It is evident from Figure 2 that Eq. 1 is useful for a description of smooth failure with a flat limit point on the stress-strain curve, which corresponds to a gradual process of the bond rupture. In the case of more abrupt bond ruptures, however, a much sharper transition to the material instability occurs. To describe such sharp transition to failure, Eq. 1 can be generalized as follows, ${ }^{50}$ for example,

$$
\psi=\frac{\Phi}{m}\left\{\Gamma\left(\frac{1}{m}, 0\right)-\Gamma\left(\frac{1}{m}, \frac{W^{m}}{\Phi^{m}}\right)\right\} .
$$

where the upper incomplete gamma function $\Gamma(s, x)=\int_{x}^{\infty} t^{s-1} \exp (-t) d t$ is used.

New parameter $m$ controls the sharpness of the transition to material instability on the stressstrain curve. By increasing/decreasing $m$, it is possible to simulate more/less steep ruptures of the internal bonds. It should not be missed that Eq. 2 reduces to Eq. 1 for $m=1$.

We note that the maximum failure energy that can be dissipated by the infinitesimal material volume is now

$$
\psi^{\text {failure }}=\frac{\Phi}{m} \Gamma\left(\frac{1}{m}, 0\right)
$$

Thus, in the cases where $m>1$, the energy limiter is not directly equal to the failure energy. For example, in the case of the abrupt rupture of the internal bonds where $m=10$, Eq. 3 takes the form $\psi^{\text {failure }}=0.95 \Phi$.

Let us apply Eq. 2 to the filled natural rubber (NR) vulcanizate with the following intact strain energy calibrated by Hamdi et al. ${ }^{52}$ :

$$
W=\sum_{k=1}^{3} C_{k}\left(\lambda_{1}^{2}+\lambda_{2}^{2}+\lambda_{3}^{2}-3\right)^{k}, \quad J=\lambda_{1} \lambda_{2} \lambda_{3}=1,
$$

where $C_{1}=0.298 \mathrm{MPa}, C_{2}=0.014 \mathrm{MPa}, C_{3}=0.00016 \mathrm{MPa}$, and $\lambda_{1}$ is a principal stretch.

First, we choose $m=10$ for the abrupt rupture of rubber.

Second, we fit the energy limiter $\Phi=82.0 \mathrm{MPa}$ to the critical failure stretch $\lambda^{c r}=7.12$ found by Hamdi et al. ${ }^{52}$ in the uniaxial tension test (Figure 3). 


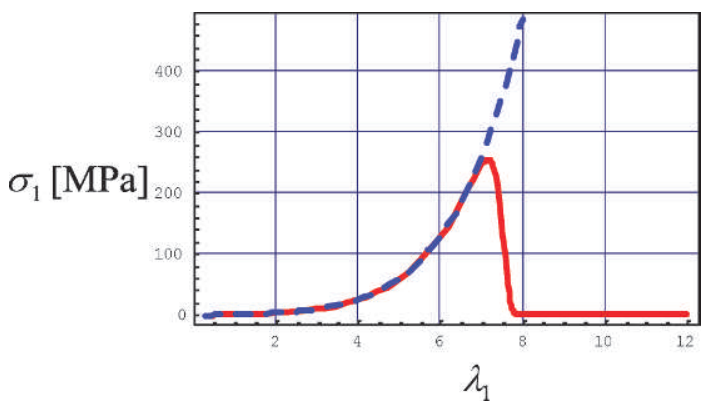

FIG. 3. - Cauchy stress versus stretch in uniaxial tension of NR: dashed line designates the intact model; solid line designates the model with energy limiter $\Phi=82.0 \mathrm{MPa}$ for $m=10$.

The calibrated strain energy function incorporating the energy limiters allows for the constitutive description of any deformation and not just the one used for calibration. Thus, it is important to examine the theory considering various stress-strain states. Unfortunately, not many experiments are reported in the literature to confront the theoretical predictions. Fortunately, Hamdi et al. ${ }^{52}$ performed a series of experiments on NR failure in biaxial tension. The comparison of the theoretical and experimental results is presented in Figure 4, where the theoretical critical points of rupture were computed from the singularity condition of the Hessian of the strain energy of $\partial^{2} \psi / \partial \lambda_{1}^{2} \cdot \partial^{2} \psi / \partial \lambda_{2}^{2}-\left(\partial^{2} \psi / \partial \lambda_{1} \partial \lambda_{2}\right)^{2}=0$.

The experimentally observed critical stretches are slightly lower than the theoretically predicted ones in the case of the equal biaxial tension because of the effects of the material and geometrical imperfections, which escape the idealized theoretical formulation. Nonetheless, the ability of the theory to predict failure seems to be encouraging.

Finalizing the description of the general idea of energy limiters, we should draw the reader's attention to the fact that the maximum strain energy density — the failure energy $\psi^{\text {failure }}$ - does not correspond to the breakage of all internal bonds. Only the weakest links break inside the infinitesimal material volume, triggering failure. We can estimate the fraction of the broken bonds. Indeed, the failure energy in the case of NR (Eq. 3) takes the value

$$
\psi^{\text {failure }}=80[\mathrm{MPa}]=80\left[\mathrm{~J} / \mathrm{cm}^{3}\right] \text {. }
$$

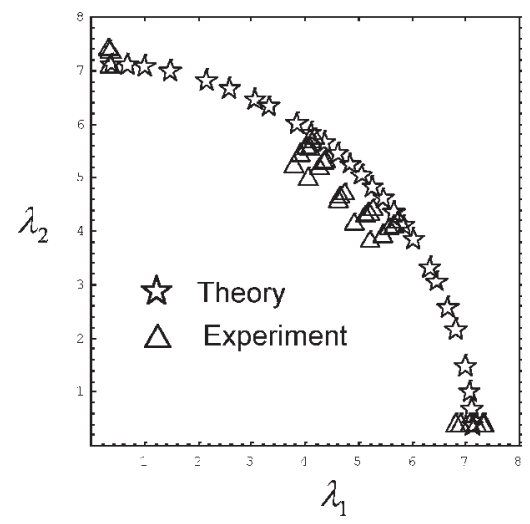

FIG. 4. - Critical failure stretches in biaxial tension for NR. 
By evaporating rubber, it is possible to find the cohesive energy (i.e., the energy of the breakage of all bonds). Treloar ${ }^{2}$ reports the following experimental value of the cohesive energy density for NR:

$$
\psi^{\text {cohesion }}=266\left[\mathrm{~J} / \mathrm{cm}^{3}\right] .
$$

Dividing Eq. 5 by Eq. 6, we get the fraction of the broken bonds:

$$
\frac{\psi^{\text {failure }}}{\psi^{\text {cohesion }}}=0.3,
$$

which means that approximately every third bond has to be broken to lead to the local failure of material.

In the subsequent sections, we apply the developed theoretical approach to modeling various failure problems.

\section{REMARK}

The calibration of energy limiters and, in a general prospect, strength of materials is based on the assumption that failure occurs homogeneously and material particles break simultaneously. The latter is an idealization that is presented by the limit/peak point on the stress-strain curve. Clearly, real materials are not ideal, and they fail nonhomogeneously depending on material features and imperfections ${ }^{53}$ of a particular specimen (by imperfections we do not mean macroscopic cracks, notches etc, which trigger the macroscopic stress concentration, rather, we mean imperfections on the size of the internal material structure). Thus, failure normally localizes into cracks. If so, the question should be asked whether the concept of material strength (or energy limiter) is physically reasonable. The answer can be found in experimental observations on failure of various specimens of the same material under similar loads. If the critical load scatters significantly for various specimens, then there is no strength of material. If the critical load does not scatter significantly and cracks appear at approximately the same load, then material has strength (and the energy limiter can be calibrated). To the best of the author's knowledge, most specimens fail at approximately the same loads for the same material, thus supporting the concept of strength. However, it is generally impossible to predict exactly where cracks will appear under the homogeneous deformation, but it is possible to predict when they will appear. Evidently, localization into cracks occurs near the peak/ limit point on the stress-strain curve, making the concept of strength of materials and energy limiters useful. Needless to say, structural design is based heavily on the concept of strength of materials, and it proved itself superbly.

\section{INFLATION AND RUPTURE OF RUBBER MEMBRANE}

The modeling of the inflation of rubber membranes is a dearly loved subject. ${ }^{51,54-63}$ The topic culminated in the recent book by Muller and Strehlow, ${ }^{64}$ which is completely devoted to rubber balloons.

However, none of the works considering the membrane deformation also addressed the failure issue. The latter has been done for the first time by Balakhovsky and Volokh, ${ }^{65}$ who used the hyperelastic model with energy limiters described above to simulate the inflation of a plane circular NR membrane of radius $1 \mathrm{~cm}$ and thickness $0.01 \mathrm{~cm}$ fixed at its edge. The pressure was increased gradually in a quasi-static mode until rupture, which occurred at a pressure of $\sim 44 \mathrm{KPa}$ in the center of the membrane, as shown in Figure 5. 


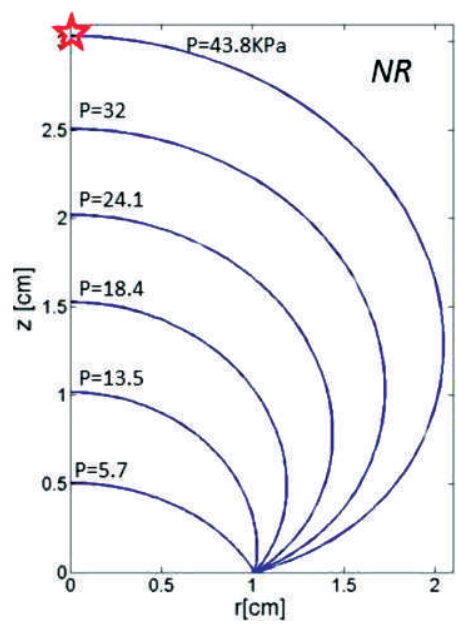

FIG. 5. - Inflation of NR membrane under the increasing hydrostatic pressure; the star designates the point of rupture. ${ }^{65}$

The point of rupture corresponded to the singularity of the structural stiffness matrix or the Hessian of the total energy of the membrane including the pressure potential. Although pressure load generally depends on displacements, in the case of the axisymmetric membrane, the pressure load is conservative. The latter remarkable result was due to work by Fried. ${ }^{60}$

It is interesting also that the point of rupture was the last one that was possible to track by using the equilibrium path-following methods. There is no further equilibrium path behind the point of rupture, and dynamics should be considered to trace the propagation of cracks.

At the center of the membrane, the equal biaxial stress-strain state was developed (Figure 6) and the critical stretches were equal to $\sim 5$ as expected from the plane biaxial analysis (Figure 4 ). It was also found that the stresses at the point of rupture (Figure 6) were essentially smaller than the rubber strength - the critical stress in the uniaxial tension tests (Figure 3). The latter finding questions the applicability of the concept of the material strength defined in uniaxial tests to the multiaxial strain-stress states. We will discuss the latter issue below.

\section{CAVITATION INSTABILITY IN RUBBER}

Unstable growth of voids or cavities is a typical mechanism of material failure. Cavitation instabilities generally occur in the material regions undergoing hydrostatic tension, and they can lead to failure localization and crack propagation. Various studies have been performed on modeling cavitation in elastic materials. ${ }^{53,66-69}$ Review articles by Gent, ${ }^{70}$ Horgan and Polignone, ${ }^{71}$ and Fond ${ }^{72}$ put special emphasis on cavitation in rubberlike materials. Although the quoted works consider mainly three-dimensional (3D) voids in the bulk material, a separate series of studies has been devoted to the instability of two-dimensional (2D) voids in thin material sheets (membranes) under the biaxial tension. ${ }^{73-75}$

Volokh ${ }^{76}$ used hyperelastic models with energy limiters described above to analyze the growth and instability of 3D voids in the bulk (Figure 7) and 2D voids in thin membranes (Figure 8) under the remote hydrostatic tension.

It was found that starting from the hydrostatic tension of $\sim 2.3 \mathrm{MPa}$ for the bulk and $\sim 56 \mathrm{MPa}$ for the membrane, the void expands unstably; it yields.

It should not be missed that the unstable yield of the void is a result of the assumption of the ideally symmetric deformation. This assumption is restrictive, of course, and it will be violated for 

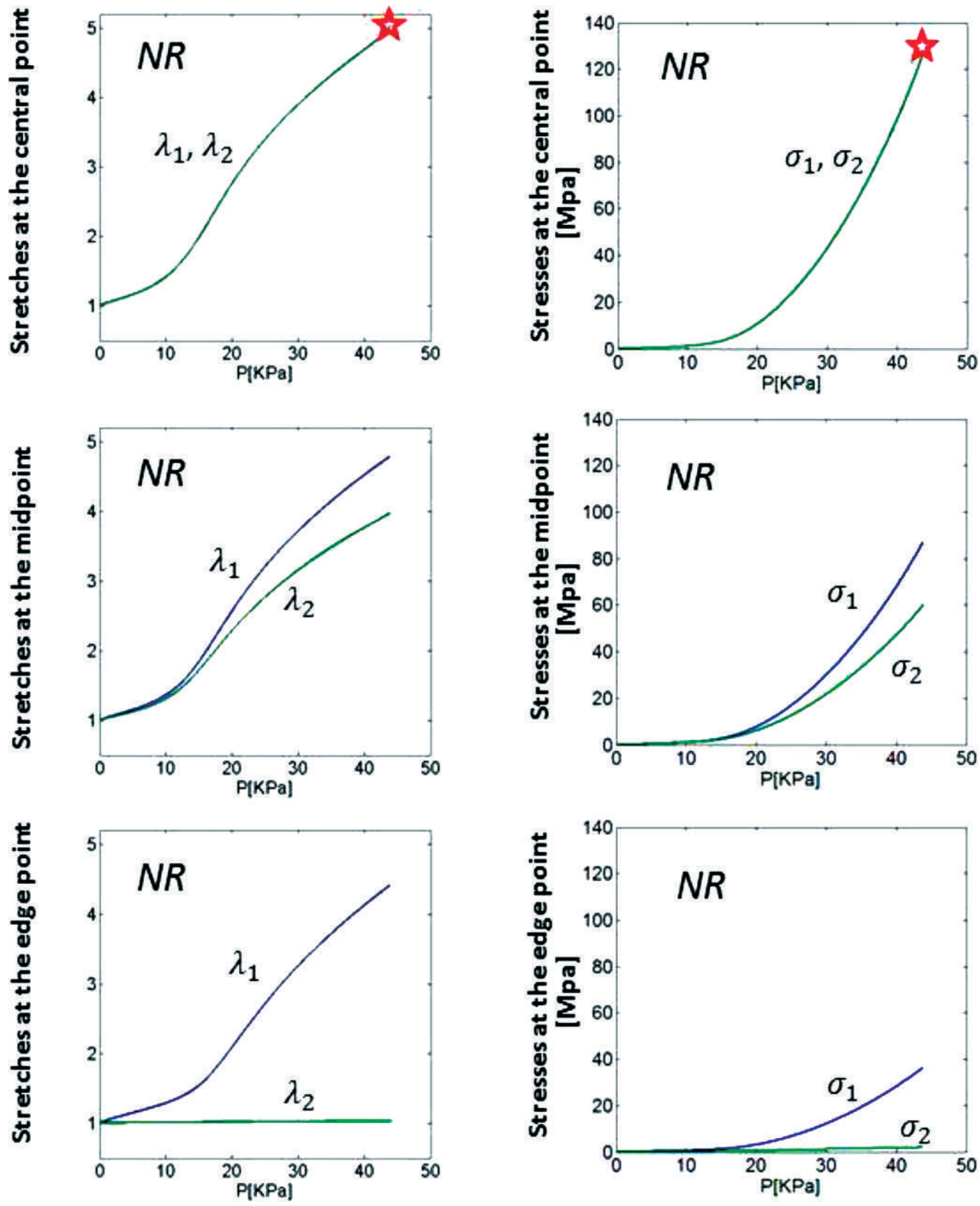

Fig. 6. - Principal stretches and stresses at the central, mid-, and edge points of the inflating membrane; stars designate points of rupture. ${ }^{65}$

real materials, which are not perfect. The latter will trigger localization of failure in the vicinity of the critical point. Nonetheless, the prediction of the critical point of the void instability seems to be reasonable even in the presence of imperfections.

The critical magnitudes of the hydrostatic tension correspond to the critical void hoop stretches equal to $\sim 5.4$ and $\sim 7.5$ for the bulk and membrane accordingly. It should not be missed, of course, 


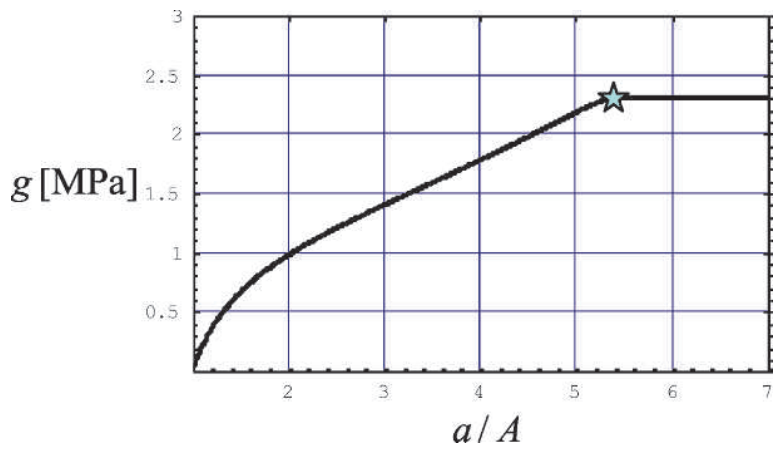

FIG. 7. - Bulk void: hydrostatic tension versus void hoop stretch for NR; the star designates the critical point of instability. ${ }^{76}$

that although the critical value of the bulk tension is smaller than the critical value of the membrane tension, the critical integral force that is imposed on the bulk void is proportional to the surface area of a 3D sphere, whereas the critical integral force that is imposed on the membrane void is proportional to the perimeter of a $2 \mathrm{D}$ circle times the membrane thickness. Consequently, the integral force on the void in the bulk will be generally greater than the integral force on the membrane void.

\section{CRACKS IN RUBBER}

The onset of the propagation of the preexisting cracks is another reason for failure of rubber structures. It was interesting, therefore, to apply the concept of the energy limiters to the study of the material and structural instability in the presence of a small crack: the Griffith problem. The latter has been done by Trapper and Volokh, ${ }^{77}$ who considered Neo-Hookean material enhanced with the energy limiter that could be derived from Eq. 2 and Eq. 4 for $m=1, C_{1}=\alpha / 2, C_{2}=0$, and $C_{3}=0$ :

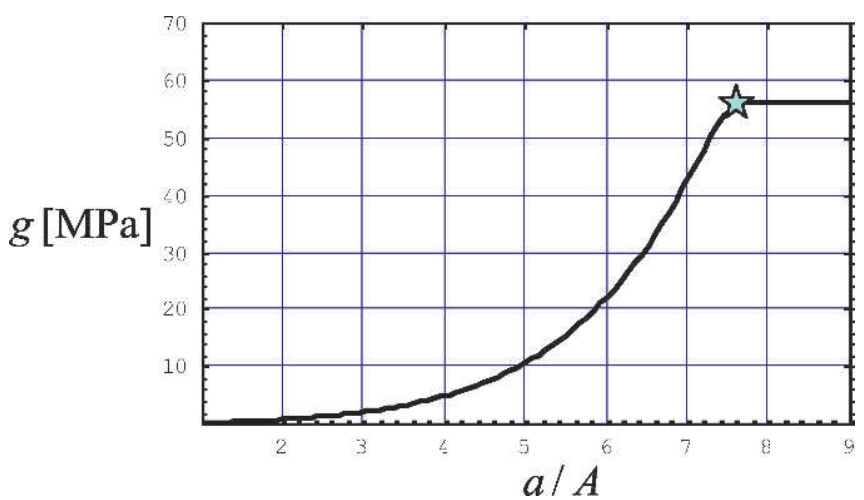

FIG. 8. - Membrane void: hydrostatic tension versus void hoop stretch for NR; the star designates the critical point of instability. ${ }^{76}$ 


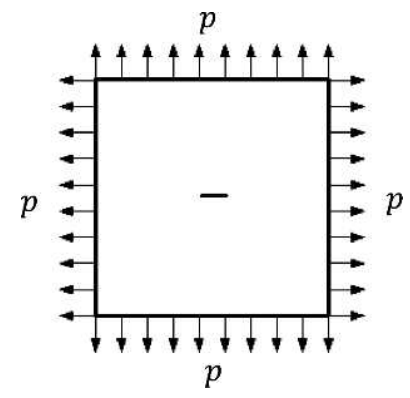

FIG. 9. - Crack in a rubber sheet under hydrostatic tension.

$$
\psi=\Phi-\Phi \exp \left\{\frac{\alpha}{2 \Phi}\left(\lambda_{1}^{2}+\lambda_{2}^{2}+\lambda_{3}^{2}-3\right)\right\}, \quad J=\lambda_{1} \lambda_{2} \lambda_{3}=1,
$$

where $\alpha$ is the shear modulus.

The purpose of the analysis was to simulate the transition to the failure propagation of a thin rubber sheet with a small crack under the hydrostatic tension (Figure 9).

For this purpose, the strain energy function with the energy limiter (Eq. 8) was plugged in ABAQUS. ${ }^{78}$ The state of the plane stress was considered for a square sheet with the varying ratio of the shear modulus to the average bond energy: $\alpha / \Phi$. To capture the stress-strain concentration at the tip of the crack, very fine meshes were used, as illustrated in Figure 10. The number of elements varied in computations to ensure convergence of the results.

The critical load of the onset of static structural (global) instability was calculated by using the ABAQUS procedure for the equilibrium path tracing. The critical load corresponds to the maximum tension that the rubber sheet can bear before the crack propagation starts.

It was observed based on numerous parametric studies that lower magnitudes of the critical tension were driven by (1) sharper cracks, (2) lengthier cracks, (3) lower ratio of the shear modulus to the average bond energy. Factors (1) and (2) directly echo the classical theories of brittle fracture. Factor (3) is more specific of soft materials undergoing large deformations. Softer materials are less sensitive to the crack sharpness because they undergo large deformations at the tip of the crack. To
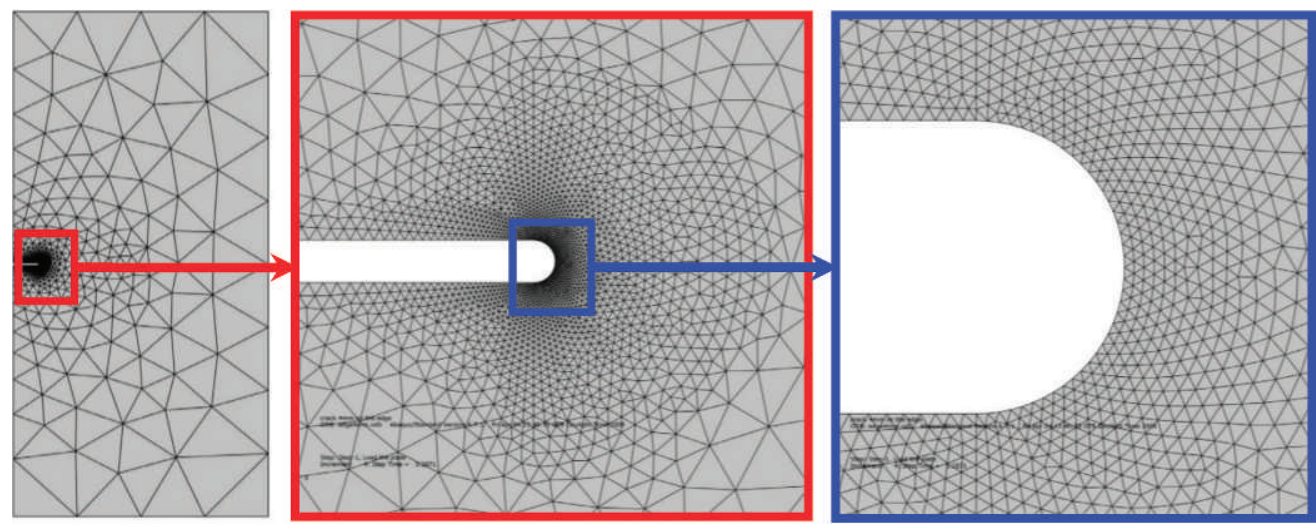

FIG. 10. — Sample finite element mesh. ${ }^{77}$ 

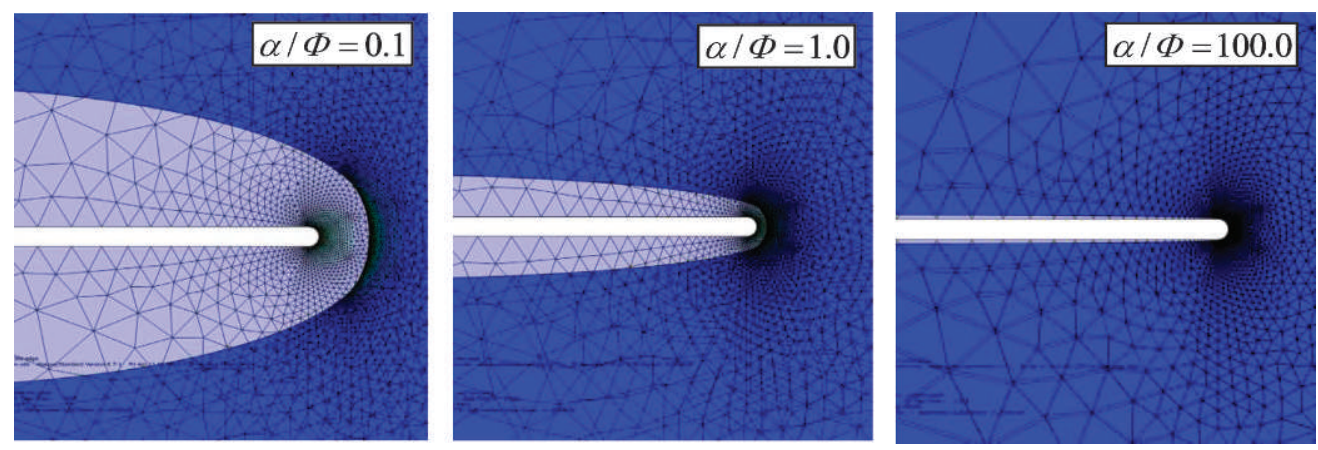

FIG. 11. - Finite element meshes at the initial (gray) and the critical (blue) states at the tip of the crack for the increasing $\alpha / \Phi$ ratio. ${ }^{77}$

illustrate this point, the finite element meshes at the beginning of loading and at the critical load are superimposed and compared in Figure 11. Evidently, huge deformations can develop around the crack for softer materials.

The observation of the role of the crack sharpness agrees well with the celebrated Inglis finding within the linear elasticity framework that the stress at the tip of an elliptic crack strongly depends on its sharpness. Assuming that the stress at the tip controls material strength, it follows that the crack sharpness affects the onset of material failure.

The observation of the role of the crack length is in partial agreement with the linear elastic fracture mechanics. The simulations of the straight cracks show that the critical tension depends approximately inversely on the square root of the crack length in full harmony with the Griffith finding. Unfortunately, that is true only for the equivalent cracks (i.e., cracks with the same tips).

The observations of the role of the $\alpha / \Phi$ ratio strongly suggest that the decrease of the shear modulus as compared with the failure energy leads to a decline of the material sensitivity to a cracklike flow. This means, specifically, that the dependence of the critical load on the crack length and sharpness is less pronounced in softer materials than in harder ones. The latter happens because softer materials can undergo large deformations suppressing the stress-strain concentration. In other words, softer materials absorb the high stresses/strains at the tip of the crack due to large deformations. To avoid confusion, however, it should be emphasized that although softer materials are less sensitive to the crack length and sharpness, they tear under lower critical loads than harder materials.

\section{ON POINTWISE FAILURE CRITERIA}

The fact that failure is incorporated in hyperelasticity with energy limiters allows for the reassessment of the pointwise failure criteria of strength of materials. Indeed, based on the presented NR model, it is possible to calculate the critical rupture states of a material sheet under the varying biaxiality parameter

$$
n=\ln \lambda_{2} / \ln \lambda_{1},
$$

where $\lambda_{1}$ and $\lambda_{2}$ are the principal stretches in the plane of the sheet.

The uniaxial tension, the pure shear, and the equal biaxial tension correspond to $n=-0.5, n=$ 0.0 , and $n=1.0$ accordingly. All of these states are homogeneous, and the failure of the ideal sheet occurs simultaneously at every point. Consistent with the used constitutive theory, the event of failure corresponds to the critical condition of the onset of the static instability and the onset of 
Maximum stretch

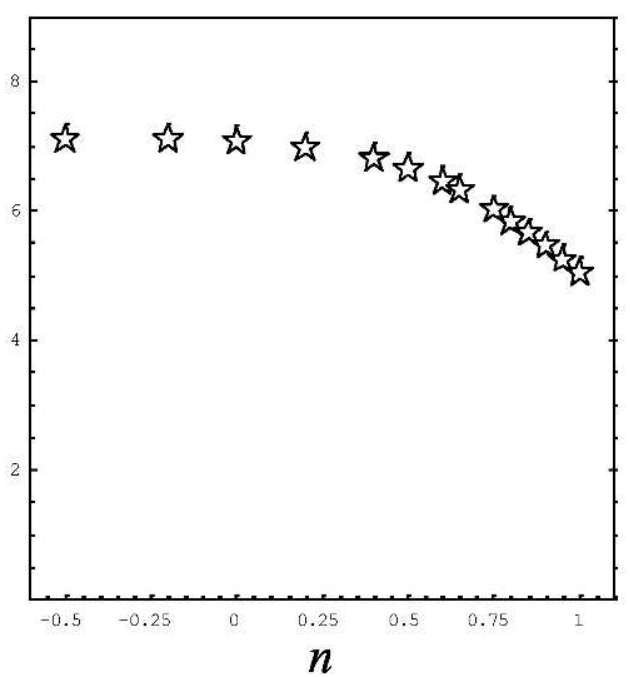

Von Mises stress $(\mathrm{MPa})$

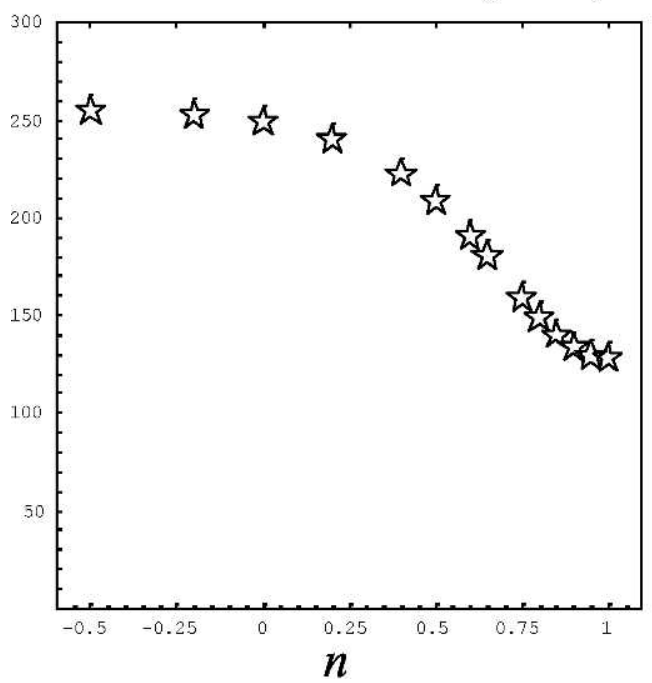

Maximum stress (MPa)

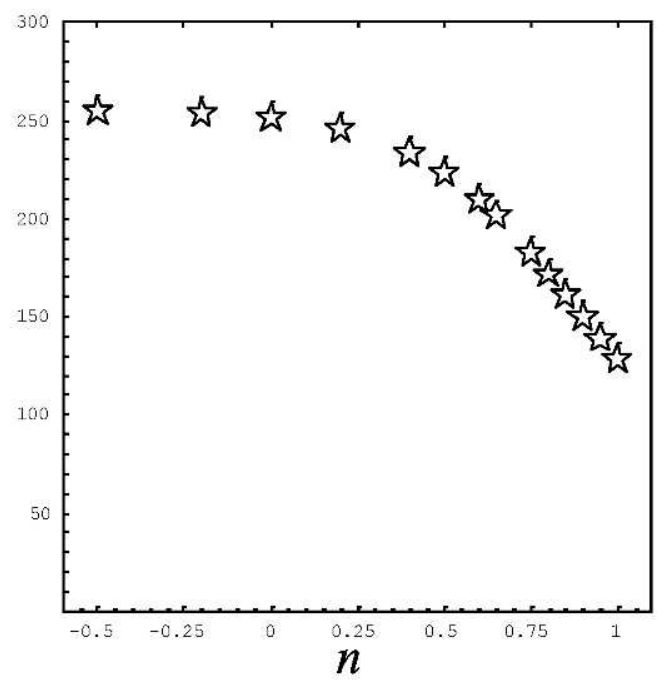

Energy (MPa)

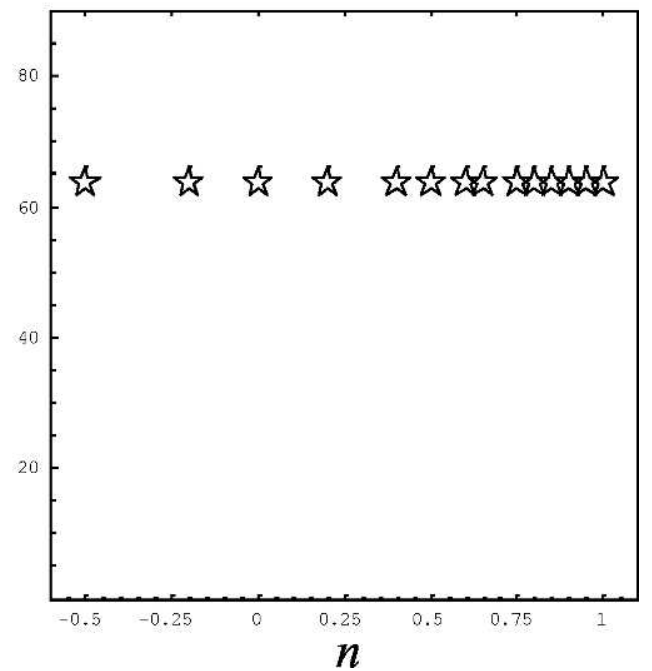

FIG. 12. - Critical failure criteria for NR under varying biaxiality ratio.

dynamic failure, where $\partial^{2} \psi / \partial \lambda_{1}^{2} \cdot \partial^{2} \psi / \partial \lambda_{2}^{2}-\left(\partial^{2} \psi / \partial \lambda_{1} \partial \lambda_{2}\right)^{2}=0$. It is possible to calculate the popular local failure criteria including maximum stresses and stretches, von Mises stress, and the energy density for the critical condition (Figure 12).

Von Mises stress presented in the figures is calculated as follows: $\sigma=$ $\sqrt{3\left(\boldsymbol{\sigma}: \boldsymbol{\sigma}-(\operatorname{tr} \boldsymbol{\sigma})^{2} / 3\right) / 2}$, where $\boldsymbol{\sigma}$ is the Cauchy stress tensor.

The results in Figure 12 demonstrate that only the energy density is almost constant for the critical failure states with varying biaxiality. It is especially remarkable that critical failure criteria corresponding to the uniaxial tension, which are usually fitted in experiments, decrease with the 


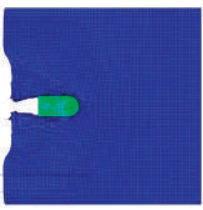

$0.2 \mathrm{~ms}$

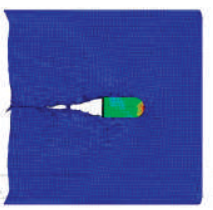

$0.4 \mathrm{~ms}$

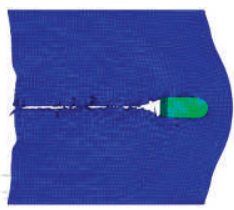

$0.6 \mathrm{~ms}$

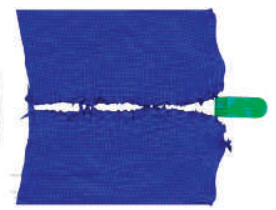

$0.8 \mathrm{~ms}$

FIG. 13. - Projectile penetration at the speed of $200 \mathrm{~m} / \mathrm{s}$ : snapshots at $0.2,0.4,0.6$, and $0.8 \mathrm{~ms} .^{80}$

developing biaxiality. Thus, the rupture under equal biaxial tension occurs under smaller values of the critical parameters (except the energy density) than is observed in the uniaxial tension. The latter notion is very important because rubbers and rubberlike materials are often loaded in the biaxial or triaxial stress-strain states where the strength criteria based on uniaxial tension tests might not be applicable.

It is worth noting here that $\mathrm{Sih}^{79}$ used the local energy in the form $S / r$ for prediction of the onset of crack propagation in brittle solids, where $S$ was the strain energy density factor and $r$ was the distance from the tip of the crack. Evidently, Sih's approach is controversial because the energy expression has singularity.

\section{DYNAMIC FAILURE IN RUBBER}

Hyperelasticity with energy limiters was used by Trapper and Volokh ${ }^{80}$ to model high-velocity penetration in a sheet of NR. Particularly, the model described by Eq. 2 and Eq. 4 was plugged in ABAQUS for the 3D explicit analysis of the edge penetration of a stiff elastic projectile into a thin sheet of the NR, and the time history of the failure process was tracked. During the computations, the failed elements were deleted based on the following criterion:

$$
\psi-\psi^{\text {failure }} \leq \text { Tolerance, }
$$

where the failure energy has been defined in Eq. 3.

Deleting the failed elements is necessary to prevent the material from healing when a returning wave of deformation can restore the failed elements. The element "killing" or removing procedure is an integral part of the commercial finite element software dealing with failure simulations. Usually, the elements are removed forcefully when a criterion of the removal is obeyed. In the case of elasticity with energy limiters, contrary to the widespread finite element technologies, there is no need to kill the elements as they die on their own, and it is only necessary to remove the failed elements.

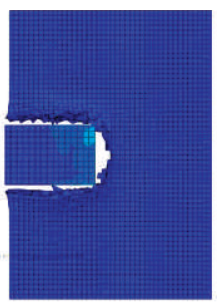

$0.02 \mathrm{~ms}$

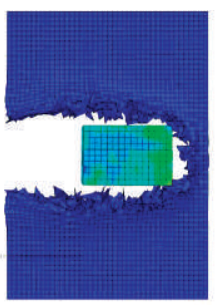

$0.04 \mathrm{~ms}$

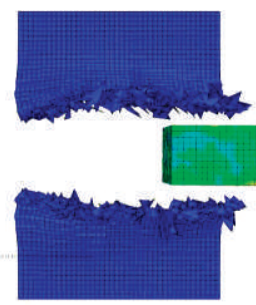

$0.06 \mathrm{~ms}$

FIG. 14. - Projectile penetration at the speed of $1500 \mathrm{~m} / \mathrm{s}$ : snapshots at $0.02,0.04$, and $0.06 \mathrm{~ms} .^{80}$ 
Two series of simulations were performed by Trapper and Volokh, ${ }^{80}$ where the reader can find the details.

First, a low-velocity penetration of a relatively sharp projectile was tracked (Figure 13). The projectile propagated by destroying a rubber layer of the height of two finite elements. In other words, the projectile propagated a crack ahead of it. The reasons for such behavior were the following: the rubber could undergo large deformations, the projectile was relatively sharp, and the velocity of the penetration was relatively low. Essentially, the observed failure propagation was analogous to cutting a rubber sheet with the help of scissors, which would also create a crack that could not propagate on its own and would require constant support from the scissors. An important observation was that failure tended to localize in a thin band, and consequently, the calculations exhibited the pathological mesh sensitivity. Indeed, the smaller the finite elements, the thinner the band.

Second, a high-velocity penetration of a flat projectile was tracked (Figure 14). Contrary to the previous simulation, however, Figure 14 shows that the projectile propagated by massively destroying finite elements ahead of the cutting edge. There was no failure localization into thin bands, and consequently, no pathological mesh sensitivity was observed.

Of course, even in the absence of sharp localizations or in the cases in which such localizations are directly enforced in analysis, high-strain gradients and bifurcation multiplicity are two main sources of mesh sensitivity. The necessity to treat high-strain gradients by refining the mesh is not specific to the material models with softening; this is the central issue of the finite element analysis as a whole. Bifurcation multiplicity is more typical of models with softening. It is worth noting that both bulk and surface (cohesive zone) models can suffer from bifurcation multiplicity. The latter may trigger some uncertainty (i.e., mesh dependency) of numerical simulations. It seems reasonable to assume that the numerical uncertainty reflects the real physical uncertainty of the problem of material failure. Unfortunately, one can regularize mathematics not physics.

\section{FAILURE LOCALIZATION IN RUBBER}

It was observed in the simulation of the dynamic failure of rubber in the previous section that material failure can localize in thin bands (cracks). This failure mode exhibits pathological mesh sensitivity in finite element simulations, and it requires regularization. A way to suppress this pathological mesh sensitivity is to enforce the characteristic length of the failure localization in the spatial discretization of material. For example, the characteristic length of the failure localization (h) can set the size of the mesh in the case of the finite elements with the linear shape functions. The fixed size of the mesh is thus physically motivated, and it should be used in the material areas where failure is supposed to localize in cracks and propagate.

The characteristic length of the failure localization has a deep physical meaning. Indeed, people used to think that cracks appear as a result of an ideal separation of two adjacent atomic layers. Although such a scenario might be reasonable for nanostructures such as graphene, carbon nanotubes, and so forth, it is unreasonable for structural materials at the macroscopic scale. In the latter case, the crack appears as a result of the massive breakage of atomic bonds. It is crucial to realize that the process of bond breakage is not confined to two neighbor atomic planes. Just the opposite: the process involves thousands of atomic planes within the representative characteristic volume of size $h$. It might be surprising at first glance, but the crack surfaces created after fracture did not need to be the closest neighbors inside the bulk before fracture.

Volokh ${ }^{81}$ proposed the following procedure for a direct calculation of the characteristic length for rubber. It assumes that the characteristic size of the representative volume where bonds break during fracture is $h$. Then, the work dissipated during the fracture process within the volume is $\omega h^{3}$, where $\omega$ is the density of the volumetric work of fracture. In the case of rubber fracture, all 
TABLE I

Comparison of Continuum Damage Mechanics AND the Methods of EnERGy Limiters

\begin{tabular}{lcccc}
\hline & $\begin{array}{c}\text { Damage } \\
\text { internal variables }\end{array}$ & $\begin{array}{c}\text { Damage } \\
\text { threshold condition }\end{array}$ & $\begin{array}{c}\text { Damage } \\
\text { evolution equation }\end{array}$ \\
\hline Damage Mechanics & Yes & Yes & Yes \\
Energy Limiters & No & No & No \\
\hline
\end{tabular}

work is consumed by the breaking bonds. On the other hand, the energy of the creation of two surfaces from the bulk is $\sim \gamma h^{2}$, where $\gamma$ is the density of the surface work of fracture introduced by Griffith. ${ }^{82}$ Equating two works, $\omega h^{3}=\gamma h^{2}$, it is possible to calculate the characteristic length of the failure localization:

$$
h=\frac{\gamma}{\omega} .
$$

In the case of NR, Rivlin and Thomas ${ }^{83}$ provided the value of the surface work of fracture - the energy of tearing - which can be used in calculations (actually, there is a range of values for the surface failure energy because of the experimental inaccuracies) ${ }^{54}$

$$
\gamma=1.3\left[\mathrm{~J} / \mathrm{cm}^{2}\right]
$$

On the other hand, the volumetric work of fracture is given in Eq. 5,

$$
\omega=\psi^{\text {failure }}=80\left[\mathrm{~J} / \mathrm{cm}^{3}\right] .
$$

Substituting Eq. 12 and Eq. 13 in Eq. 11, it is possible to find the characteristic length

$$
h \approx 0.2[\mathrm{~mm}] .
$$

The obtained characteristic length can be tackled in computations. However, this small size is only crucial for the finite elements in the areas where the crack propagation takes place. Nonfractured areas can be approximated arbitrarily.

It is very important to emphasize that according to the present approach, both volumetric, $\omega$, and surface, $\gamma$, works of fracture are assumed to be material parameters, and thus, the characteristic length of the failure localization is a material parameter too. In contrast to the approach described above, some authors suggest that the volumetric work of fracture is not a material parameter but a variable that should be adjusted to the size of the mesh of the spatial discretization. In the latter case, the constitutive law depends on the discretization procedure violating the physical approach of continuum mechanics.

It is quite amazing that the experiments required for the calculation are macroscopic whereas the characteristic length is an internal structural parameter of the material. It should not be missed, however, that the experimental data used for the calculation of the characteristic length were obtained under the quasi-static loads, whereas during the dynamic crack propagation, both values of the surface and volumetric fracture work can alter, leading to the possible alteration of the characteristic length.

\section{CONCLUDING REMARKS}

Traditional hyperelastic models of rubber and rubberlike materials allow accumulating strain energy unlimitedly. The latter is unphysical, and the possibility of material failure should be 
included in the theoretical description. A way to describe failure is to introduce energy limiters in the expression of the strain energy. This way is essentially an extension of the description of a twoparticle separation to large amounts of particles (continuum). The methods of elasticity with energy limiters are dramatically simpler than the existing methods of CMD, for example, that are frequently used for modeling bulk failure (Table I).

In the present work, we reviewed some applications of the elasticity with energy limiters or softening hyperelasticity to the classical problems in mechanics of rubber, which included the inflation and rupture of rubber membranes, 2D and 3D cavitation instabilities under the hydrostatic pressure, the onset of the propagation of the preexisting small cracks, the reassessment of the strength-of-materials local failure criteria, dynamic failure propagation triggered by the penetration of a projectile, and the calculation of the characteristic length of failure localization in rubber.

There are some interesting topics beyond the reviewed works. For example, an extension of the elasticity with the energy limiters to the case of the viscoelastic response was considered by Volokh and Trapper. ${ }^{84}$ An extension of the elasticity with the energy limiters to the modeling of failure of anisotropic rubberlike soft tissues was considered by Volokh. ${ }^{85}$ It is also of interest to attempt describing the Mullins effect with the help of the energy limiters. The latter will require a consideration of the different families of material bonds and corresponding different families of the energy limiters. There are quite a few experiments in which the crack propagation in rubber was traced, ${ }^{86,87}$ their numerical simulation is desirable as well.

Another focus of the current studies in rubber failure, which was not discussed in the present work, is related to the multiaxial fatigue problems, and there may be important connections between the energy limiters theory and the critical plane theories. The main idea in the latter approaches is that the forces driving failure are evaluated for each possible failure plane of the material, in order to identify the plane on which the most damaging forces occur. ${ }^{88-95}$

Concerning the limitations of the energy limiters approach, we should mention that more experimental data are needed for the general validation and possible revision of the theory. Such revisions might be necessary for dynamic problems, fatigue, cases in which a significant amount of energy can be stored in ways that do not contribute to crack growth, and so forth. We hope, nonetheless, that the simple yet deep physical roots of the approach of energy limiters may be appealing for the practical analysts and designers of rubber elements and structures.

\section{REFERENCES}

${ }^{1}$ G. I. Barenblatt and D. D. Joseph, Collected Papers of R. S. Rivlin, Springer, New York, 1996.

${ }^{2}$ L. R. G. Treloar, The Physics of Rubber Elasticity, Oxford University Press, Oxford, 1975.

${ }^{3}$ M. C. Boyce and E. M. Arruda, RubBer Chem. TeChNOL. 73, 504 (2000).

${ }^{4}$ J. K. Knowles and E. Sternberg, J. Elasticity 3, 67 (1973).

${ }^{5}$ J. K. Knowles, Int. J. Fract. 13, 611 (1977).

${ }^{6}$ J. M. Herrmann, J. Elasticity 21, 227 (1989).

${ }^{7}$ P. H. Geubelle and W. G. Knauss, J. Elasticity 35, 61 (1994).

${ }^{8}$ P. H. Geubelle, Int. J. Solids Struct. 32, 1003 (1995).

${ }^{9}$ E. Verron, RubBer CHEM. TECHNOL. 83, 270 (2010).

${ }^{10}$ B. N. Persson, O. Albohr, G. Heinrich, and H. Ueba, J. Phys. Cond. Matter 17, R1071 (2005).

${ }^{11}$ A. N. Gent, Engineering with Rubber: How to Design Rubber Components, Hanser, Munich, 2001.

${ }^{12}$ E. Bouchbinder, Phys. Rev. Lett. 103, 164301 (2009).

${ }^{13}$ A. Livne, E. Bouchbinder, I. Svetlizky, and J. Fineberg, Science 327, 1359 (2010).

${ }^{14}$ M. Marder, Phys. Rev. Lett. 94, 048001 (2005). 
${ }^{15}$ M. Marder, J. Mech. Phys. Solids 54, 491 (2006).

${ }^{16}$ G. I. Barenblatt, J. Appl. Math. Mech. 23, 622 (1959).

${ }^{17}$ A. Needleman, J. Appl. Mech. 54, 525 (1987).

${ }^{18}$ J. R. Rice and J. S. Wang, Mater. Sci. Eng. A 107, 23 (1989).

${ }^{19}$ V. Tvergaard and J. W. Hutchinson, J. Mech. Phys. Solids 40, 1377 (1992).

${ }^{20}$ G. T. Camacho and M. Ortiz, Int. J. Solids Struct. 33, 2899 (1996).

${ }^{21}$ M. R. Gurvich, RubBer Chem. TeCHnOl. 84, 354 (2011).

${ }^{22}$ R. De Borst, Int. J. Numer. Meth. Eng. 52, 63 (2001).

${ }^{23}$ X. P. Xu and A. Needleman, J. Mech. Phys. Solids 42, 1397 (1994).

${ }^{24}$ N. Moes, J. Dolbow, and T. Belytschko, Int. J. Num. Meth. Eng. 46, 131 (1999).

${ }^{25}$ L. M. Kachanov, Izvestiia Akademii Nauk SSSR, Otd. Teckh. Nauk 8, 26 (1958).

${ }^{26}$ S. Govindjee and J. C. Simo, J. Mech. Phys. Solids 39, 87 (1991).

${ }^{27}$ S. Govindjee and J. C. Simo, J. Mech. Phys. Solids 40, 213 (1992).

${ }^{28}$ M. A. Johnson and M. F. Beatty, Continuum Mech. Therm. 5, 83 (1993).

${ }^{29}$ M. A. Johnson and M. F. Beatty, Continuum Mech. Therm. 5, 301 (1993).

${ }^{30}$ C. Miehe, Eur. J. Mech. A Solids 14, 697 (1995).

${ }^{31}$ A. Lion, Continuum Mech. Thermodyn. 8, 153 (1996).

${ }^{32}$ H. E. Huntley, A. S. Wineman, and K. R. Rajagopal, IMA J. Appl. Math. 59, 309 (1997).

${ }^{33}$ R. W. Ogden and D. G. Roxburgh, Proc. R. Soc. Lond. A 455, 2861 (1999).

${ }^{34}$ A. D. Drozdov and A. Dorfmann, Continuum Mech. Therm. 13, 183 (2001).

${ }^{35}$ C. O. Horgan, R. W. Ogden, and G. Saccomandi, Proc. R. Soc. A 460, 1737 (2004).

${ }^{36}$ R. Dargazany and M. Itskov, Int. J. Solids Struct. 46, 2967 (2009).

${ }^{37}$ A. Elias-Zuniga and C. A. Rodriguez, Int. J. Eng. Sci. 48, 1937 (2010).

${ }^{38}$ M. Itskov, A. E. Ehret, R. Kazakeviciute-Makovska, and G. W. Weinhold, ZAMM 90, 370 (2010).

${ }^{39}$ J. Lemaitre and R. Desmorat, Engineering Damage Mechanics: Ductile, Creep, Fatigue and Brittle Failures, Springer, Berlin, 2005.

${ }^{40}$ G. Pijaudier-Cabot and Z. P. Bazant, J. Eng. Mech. 113, 1512 (1987).

${ }^{41}$ D. Lasry and T. Belytschko, Int. J. Solids Struct. 24, 581 (1988).

${ }^{42}$ H. Gao and P. Klein, J. Mech. Phys. Solids 46, 187 (1998).

${ }^{43}$ P. Klein and H. Gao, Eng. Fract. Mech. 61, 21 (1998).

${ }^{44}$ H. Dal and M. Kaliske, J. Mech. Phys. Solids 57, 1340 (2009).

${ }^{45}$ K. Y. Volokh and D. A. Vorp, J. Biomech. 41, 1015 (2008).

${ }^{46}$ M. F. Beatty, App. Mech. Rev. 40, 1699 (1987).

${ }^{47}$ K. Y. Volokh, J. Appl. Mech. 71, 141 (2004).

${ }^{48}$ K. Y. Volokh, J. Mech. Phys. Solids 55, 2237 (2007).

${ }^{49}$ K. Y. Volokh, Int. J. Multiscale Comp. Eng. 6, 393 (2008).

${ }^{50}$ K. Y. Volokh, Mech. Res. Comm. 37, 684 (2010).

${ }^{51}$ E. Verron and G. Marckmann, Thin-Walled Struct. 41, 731 (2003).

${ }^{52}$ A. Hamdi, M. Nait Abdelaziz, N. Ait Hocine, P. Heuillet, and N. Benseddiq, Polym. Test. 25, 994 (2006).

${ }^{53}$ R. Abeyaratne and C. O. Horgan, J. Elasticity 15, 243 (1985).

${ }^{54}$ J. E. Adkins and R. S. Rivlin, Phil. Trans. R. Soc. A 244, 505 (1952).

${ }^{55}$ H. O. Foster, Int. J. Eng. Sci. 5, 95 (1967).

${ }^{56}$ L. J. Hart-Smith and J. D. C. Crisp, Int. J. Eng. Sci. 5, 1 (1967).

${ }^{57}$ J. T. Oden and T. Sato, Int. J. Solids Struct. 3, 471 (1967).

${ }^{58}$ J. T. Tielking and W. W. Feng, J. Appl. Mech. 41, 491 (1974). 
${ }^{59}$ A. Needleman, Int. J. Solids Struct. 13, 409 (1977).

${ }^{60}$ I. Fried, Int. J. Num. Meth. Eng. 18, 653 (1982).

${ }^{61}$ A. Kelkar, W. Elber, and I. S. Raju, Comput. Struct. 21, 413 (1985).

${ }^{62}$ H. J. Weinitschke, Math. Meth. Appl. Sci. 9, 76 (1987).

${ }^{63}$ Y. Li, J. A. Nemes, and A. A. Derdouri, Polym. Eng. Sci. 41, 1399 (2001).

${ }^{64}$ I. Muller and P. Strehlow, Rubber and Rubber Balloons: Paradigms of Thermodynamics, Springer, Berlin, 2004.

${ }^{65}$ K. Balakhovsky and K. Y. Volokh, Int. J. Fract. 177, 179 (2012).

${ }^{66}$ M. L. Williams and R. A. Schapery, Int. J. Fract. 1, 64 (1965).

${ }^{67}$ J. M. Ball, Phil. Trans. R Soc. Lond. A 306, 557 (1982).

${ }^{68}$ O. Lopez-Pamies, J. Elasticity 94, 115 (2009).

${ }^{69}$ D. Henao, J. Elasticity 94, 55 (2009).

${ }^{70}$ A. N. Gent, RubBer ChEM. TeChNOL. 63, G49 (1990).

${ }^{71}$ C. O. Horgan and D. A. Polignone, App. Mech. Rev. 48, 471 (1995).

${ }^{72}$ C. Fond, J. Polym. Sci. B Polym. Phys. 39, 2081 (2001).

${ }^{73}$ D. M. Haughton, Q. J. Mech. Appl. Math. 39, 289 (1986).

${ }^{74}$ D. M. Haughton, Int. J. Eng. Sci. 28, 163 (1990).

${ }^{75}$ T. Cohen and D. Durban, Int. J. Eng. Sci. 48, 52 (2010).

${ }^{76}$ K. Y. Volokh, Int. J. Appl. Mech. 3, 299 (2011).

${ }^{77}$ P. Trapper and K. Y. Volokh, Int. J. Solids Struct. 45, 6034 (2008).

78“ABAQUS," Version 6.7-1, H.K.S Inc., Pawtucket, RI, 2007.

${ }^{79}$ G. C. Sih, Int. J. Fract. 10, 305 (1974).

${ }^{80} \mathrm{P}$. Trapper and K. Y. Volokh, Int. J. Fract. 162, 245 (2010).

${ }^{81}$ K. Y. Volokh, Int. J. Fract. 168, 113 (2011).

${ }^{82}$ A. A. Griffith, Phil. Trans. R Soc. Lond. A221, 163 (1921).

${ }^{83}$ R. S. Rivlin and A. G. Thomas, J. Polym. Sci. 10, 291 (1953).

${ }^{84}$ K. Y. Volokh and P. Trapper, J. Mech. Mater. Struct. 3, 1695 (2008).

${ }^{85}$ K. Y. Volokh, J. Mech. Behav. Biomed. Materials 4, 1582 (2011).

${ }^{86}$ R. D. Deegan, P. J. Petersan, M. Marder, and H. L. Swinney, Phys. Rev. Lett. 88, 014304 (2002).

${ }^{87}$ P. J. Petersan, R. D. Deegan, M. Marder, and H. L. Swinney, Phys. Rev. Lett. 93, 015504 (2004).

${ }^{88}$ W. V. Mars, RubBer CHEM. TECHNOL. 75, 1 (2002).

${ }^{89}$ W. V. Mars and A. Fatemi, J. Mater. Sci. 41, 7324 (2006).

${ }^{90}$ W. V. Mars and A. Fatemi, RubBer Chem. TeChnOL. 79, 589 (2006).

${ }^{91}$ N. Saintier, G. Cailletaud, and R. Piques, Int. J. Fatigue 28, 61 (2006).

${ }^{92}$ N. Saintier, G. Cailletaud, and R. Piques, Int. J. Fatigue 28, 530 (2006).

${ }^{93}$ R. J. Harbour, A. Fatemi, and W. V. Mars, Int. J. Fatigue 30, 1231 (2008).

${ }^{94}$ N. Andriyana, Saintier, and E. Verron, Int. J. Fatigue 32, 1627 (2010).

${ }^{95}$ G. Ayoub, M. Nait-Abdelaziz, F. Zairi, J. M. Gloaguen, and P. Charrier, Mech. Mater. 52, 87 (2012). 